\title{
Dietary patterns and their associations with childhood obesity in China
}

\author{
Jiguo Zhang ${ }^{1}$, Huijun Wang ${ }^{1}$, Youfa Wang ${ }^{2}$, Hong Xue ${ }^{2}$, Zhihong Wang ${ }^{1}$, Wenwen Du ${ }^{1}$, Chang Su ${ }^{1}$, \\ Ji Zhang ${ }^{1}$, Hongru Jiang ${ }^{1}$, Fengying Zhai ${ }^{1}$ and Bing Zhang ${ }^{1 *}$ \\ ${ }^{1}$ National Institute for Nutrition and Health, Chinese Center for Disease Control and Prevention, No. 29 Nanwei Road, \\ Beijing 100050, People's Republic of China \\ ${ }^{2}$ Department of Epidemiology and Environmental Health, School of Public Health and Health Professions, University at \\ Buffalo, State University of New York, Buffalo, NY 14214-8001, USA
}

(Submitted 22 October 2014 - Final revision received 6 February 2015 - Accepted 12 March 2015 - First published online 6 May 2015)

\begin{abstract}
Dietary patterns represent the combined effects of foods, and illustrate efficaciously the impact of diet on health outcomes. Some findings of previous studies have limited applicability to Chinese children due to cultural factors. The presnt study was designed to identify dietary patterns and determine their relationships with obesity among Chinese children and adolescents. Data collected from 1282 children and adolescents aged 7-17 years from the 2011 China Health and Nutrition Survey (CHNS) were used. Dietary patterns were identified using factor analysis of data from three consecutive 24-h dietary recalls. Weight and height were measured following standard methods, and BMI was calculated. Three dietary patterns were identified: modern (high intakes of milk, fast foods and eggs), traditional north (high intakes of wheat, tubers and other cereals) and traditional south (high intakes of vegetables, rice and pork). After adjusting for some confounders and total energy intake, subjects in the highest quartiles of the modern and traditional north patterns were found to have significantly greater risk of obesity (OR $3 \cdot 10,95 \%$ CI 1.52, 6.32, and OR 2.42, $95 \%$ CI 1.34, 4.39, respectively). In conclusion, the modern dietary pattern and the traditional north dietary pattern were associated with higher risk of obesity. Promoting healthier eating patterns could help prevent obesity in Chinese children.
\end{abstract}

Key words: Dietary patterns: Factor analysis: Obesity: Childhood

Dietary intakes during childhood may have many short- and long-term impacts on health, including obesity risks during childhood $^{(1,2)}$. Childhood obesity is a serious public health problem that needs to be addressed urgently ${ }^{(3)}$, because it leads to several medical and psychosocial health problems in children ${ }^{(2,4)}$. Moreover, overweight/obesity developed during childhood will continue into adulthood, and will be associated with risks for other diseases such as diabetes, arterial hypertension, coronary artery disease, and fatty liver disease $^{(5)}$. At least one factor in the tracking of obesity from childhood into adulthood may be the fact that some eating habits developed during childhood eventually become lifelong dietary habits ${ }^{(6)}$.

Obesity is caused by a set of complex interactions between the environment, genetic predisposition, and human behaviour $^{(4)}$. Among them, diet is an important factor. Although dietary factors have been implicated in the development of obesity, this relationship is complex and poorly understood ${ }^{(7)}$. Traditional dietary analyses have focused merely on the relationship between individual nutrients or foods and obesity, which has certain limitations ${ }^{(8-11)}$. Dietary pattern analysis has emerged as an alternative, holistic dietary approach ${ }^{(12)}$. Dietary patterns may be more informative for investigating diet-disease relationships than consumption of individual foods or nutrients, as they consider total dietary intake and the co-linearity between many foods and nutrients, as well as the potentially synergistic effects of foods and nutrients $^{(11-14)}$.

As in many other countries, nutrition transition is an ongoing phenomenon in China ${ }^{(15)}$. For Chinese children and adolescents, urbanisation and modernization have resulted in improvement in nutritional status, with declines in underweight and stunting ${ }^{(16,17)}$. But China faces a significant and rapidly growing epidemic of overweight/obesity in children and adolescents ${ }^{(18,19)}$. Several studies have shown associations of childhood obesity with specific dietary patterns such as western eating patterns with increased consumption of sweet foods, animal foods, snacks, low-fibre and high-fat diets and dieting, although the results have been inconsistent ${ }^{(20-24)}$.

Abbreviation: CHNS, China Health and Nutrition Survey.

*Corresponding author: B. Zhang, fax +86 10 83132909, email zzhangb327@aliyun.com 
But some of these findings may not especially be applicable to Chinese children due to cultural factors affecting intakes.

To fill such gaps in the literature, the present study aimed to identify the prevailing dietary patterns in Chinese children and their associations with childhood obesity.

\section{Methods \\ Study population}

We used for the present investigation the data of the China Health and Nutrition Survey (CHNS) 1989-2011 that was designed to examine how the social and economic transformation in China has affected the health and nutritional status of the Chinese population ${ }^{(25)}$. The CHNS covers twelve provinces that vary in demography, geography, economic development, and public resources. A multistage, random cluster approach was used to draw the sample surveyed in each of the provinces. The present analysis was confined to the survey conducted in 2011. Participants with energy intake $33 \mathrm{MJ} / \mathrm{d}$ (>8000 kcal/d) or $17 \mathrm{MJ} / \mathrm{d}(<400 \mathrm{kcal} / \mathrm{d})$ were excluded from the analysis. There were 1282 children and adolescents (boys $=645$, girls $=637$ ) aged 7 to 17 years with complete demographic and dietary data. All subjects' parents gave written informed consent for their children's participation in the survey. The study was approved by the Institutional Review Committees of the University of North Carolina at Chapel Hill and the National Institute of Nutrition and Food Safety, Chinese Center for Disease Control and Prevention.

\section{Measures of dietary intakes}

Dietary intake at the individual level was assessed by using three consecutive 24-h dietary recalls (two weekdays and one weekend day) in each wave of the CHNS. Interviewers were trained on using standard forms for administering the 24-h dietary recalls with food models and picture aids in the household interview. The participants, except children aged younger than 12 years, were asked to report the kinds and amounts of food and beverage items that they consumed both at home and away from home on a 24-h recall basis. For children younger than 12 years, the mother or a mother substitute who handled food preparation and feeding in the household was asked to recall the children's food consumption $^{(26)}$. The average intakes of the three $24-\mathrm{h}$ recalls were used for each individual.

\section{Other relevant variables}

Children' sex, age, urban-rural status, and annual household income were the other details collected through the use of a general information quetionnnaire with the help of mother or a mother substitute. Anthropometrical measurements were conducted by well-trained health workers who followed a reference protocol recommended by the $\mathrm{WHO}^{(27)}$. BMI was calculated using height and weight measures. Considering the differences in body composition across different ethnic groups, the Working Group for Obesity in China organized by the International Life Science Institute Focal Point in China conducted an analysis of BMI of children and adolescents aged 7-18 years. A new BMI classification reference was recommended by the Working Group for Obesity in China in 2004. Overweight was defined as BMI $>85$ th percentile but $\leq 95$ th percentile, relative to sex and age, whereas obesity was defined as BMI $>95$ th percentile ${ }^{(28)}$. This standard is the most appropriate one and has been applied extensively in recent years.

\section{Statistical analysis}

First, we conducted factor analysis (principal component) to derive food patterns based on the twenty food groups from the China Food Composition data (Appendix 1). Mean intake $(\mathrm{g} / \mathrm{d})$ was used as the input value in the analysis. The factors were rotated by an orthogonal transformation (Varimax rotation function in SAS) to achieve a more simplistic structure with greater interpretability. In considering the number of factors to retain, we evaluated eigenvalues $(>1)$, the scree plots, and the interpretability of the factors to determine which set of factors can most meaningfully describe distinct food patterns. From these analyses, the three-factor solution was selected. Items were retained in a factor if they had an absolute correlation $\geq 0.25$ with that factor. Factor loadings were calculated for each food group across the three factors. A factor score was calculated for each subject for each of the three factors, in which intakes of twenty food groups were weighted by their factor loadings and summed ${ }^{(29)}$. Quartiles were categorized across the score of each dietary pattern based on the distribution in the whole population, and used for comparison of nutrient intake and other lifestyle factors.

Logistic regression analysis was used to assess the association between obesity risks and identified dietary patterns. Multivariate adjusted OR were calculated by adjusting for potential risk factors of obesity including age, sex, living area, physical activity, annual household income, and total energy intake. The linear trend of association was assessed by a logistic regression model assigning median scores to each quartile for each dietary pattern. We also fit linear regression analysis to test the association between the dietary pattern scores and BMI.

All statistical analyses were performed using the SAS software package (version 9.2; SAS Institute, Inc.). Statistical significance was defined as $P<0.05$.

\section{Results}

\section{Dietary patterns}

Factor analysis revealed three dietary patterns: modern, traditional north and traditional south. The factor loadings of each pattern after orthogonal rotation are shown in Table 1. These three factors explained $27 \cdot 6 \%$ of the variance in total food intake. The modern pattern (Factor 1) was highly correlated with intake of milk, fast foods, eggs, other livestock meat, poultry, and cake. The traditional north pattern (Factor 2) was characterized by high intakes of wheat flour 
Table 1. Factor-loading matrix for the three dietary patterns and their food or food groups in Chinese children*

\begin{tabular}{|c|c|c|c|c|c|c|}
\hline & \multicolumn{2}{|l|}{ Modern } & \multicolumn{2}{|c|}{ Traditional north } & \multicolumn{2}{|c|}{ Traditional south } \\
\hline & Milk & 0.57904 & Wheat & 0.80593 & Vegetables & 0.74552 \\
\hline & Fast foods & 0.57151 & Other cereals & 0.66832 & Rice & 0.55451 \\
\hline & Eggs & 0.50842 & Tubers & 0.35174 & Pork & 0.40992 \\
\hline & Other livestock meat & 0.44928 & Poultry & -0.25801 & Legumes & 0.36816 \\
\hline & Poultry & 0.38905 & Pork & -0.37309 & Fungi and algae & 0.25223 \\
\hline & Cake & 0.35040 & Rice & -0.39496 & Fast foods & -0.26867 \\
\hline & Fruits & 0.34797 & & & & \\
\hline & Aquatic products & 0.32774 & & & & \\
\hline & Fungi and algae & 0.30501 & & & & \\
\hline & Beverages & 0.30497 & & & & \\
\hline & Sugary foods & 0.29213 & & & & \\
\hline & Organ meat & 0.27581 & & & & \\
\hline & Rice & -0.31451 & & & & \\
\hline$\%$ & $11 \cdot 3$ & & 9 & & 6.9 & \\
\hline
\end{tabular}

${ }^{*}$ Absolute values $<0.25$ are not presented in the table.

products and other cereals, combined with low consumption of rice, pork, and poultry, and represented atypical traditional diet in the north of China. The third factor, the traditional south pattern, represented a typical traditional diet in the south of China, characterized by high intakes of vegetables, rice, pork, and legumes.

\section{Association of dietary patterns with sociodemographic characteristics}

The characteristics of Chinese children and adolescents across quartiles of the dietary pattern scores are presented in Table 2. Two of the three dietary patterns were associated with the participants' age. Children with high scores for the modern and southern patterns $(P<0 \cdot 0001)$ were older. The modern pattern and southern pattern showed sex differences, with male subjects more likely to have high scores. Children with high scores for the modern pattern were more likely to be living in urban areas. Children with high scores for the northern pattern were more likely to be obese. Overweight and obesity increased across the quartile categories of the modern pattern $(P=0.0002,0.0005)$. However, no association was found between the southern pattern and the prevalence of overweight and obesity.

\section{Association of dietary patterns with nutrient intakes}

Children with higher scores for the modern pattern had higher intakes of energy and a higher percent of energy from fats, fibre, Ca and Fe (Table 3). Higher scores for the northern pattern were associated with higher intakes of percent energy from carbohydrates, vitamin C, fibre and Fe, and lower intakes of percent energy from fats and vitamin A. Higher scores for the southern pattern were associated with higher intakes of energy, Fe, vitamin A, vitamin C, fibre and $\mathrm{Ca}$, as well as lower intakes of percent energy from fats.

\section{Association of dietary patterns with BMI}

In the modern pattern, participants in the upper quartile had a significant increase in BMI $(\beta=1.60862,95 \%$ CI 1.04172 ,
2.17552; $P$ for trend $<0.0001$ ) (Table 4). In the traditional north pattern, participants in the upper quartile had an increase in BMI ( $\beta=1.08382,95 \%$ CI 0.55900, 1.60863; $P$ for trend $<0 \cdot 0001$ ) when we used dietary pattern scores as categorical variables (quartiles) in the multivariate linear regression models.

\section{Association of dietary patterns with childhood obesity}

Regression results of the association between the dietary patterns and obesity are shown in Table 5. After adjusting

Table 2. Characteristics of Chinese children and adolescents according to quartiles $(\mathrm{Q})$ of the three dietary patterns in China

(Mean values and standard deviations, and percentages)

\begin{tabular}{|c|c|c|c|c|c|}
\hline & \multicolumn{5}{|c|}{ Quartile of dietary pattern score } \\
\hline & Q1 & Q2 & Q3 & Q4 & $P$ for trend ${ }^{*}$ \\
\hline$n$ & 320 & 321 & 321 & 320 & \\
\hline \multicolumn{6}{|l|}{ Modern } \\
\hline Age (years) & & & & & $<0.0001$ \\
\hline Mean & $10 \cdot 9$ & $11 \cdot 0$ & $11 \cdot 2$ & $12 \cdot 0$ & \\
\hline SD & $2 \cdot 8$ & $2 \cdot 9$ & $3 \cdot 0$ & $3 \cdot 0$ & \\
\hline Female (\%) & $58 \cdot 1$ & 49.8 & $43 \cdot 3$ & 47.5 & 0.005 \\
\hline Urban (\%) & $9 \cdot 7$ & 28.7 & $37 \cdot 1$ & $47 \cdot 2$ & $<0.0001$ \\
\hline Overweight (\%) & $5 \cdot 6$ & $9 \cdot 3$ & $13 \cdot 1$ & $14 \cdot 1$ & 0.0002 \\
\hline Obesity (\%) & 4.4 & $8 \cdot 1$ & $8 \cdot 7$ & $12 \cdot 2$ & 0.0005 \\
\hline \multicolumn{6}{|l|}{ Traditional north } \\
\hline Age (years) & & & & & 0.9781 \\
\hline Mean & 11.4 & $11 \cdot 1$ & $11 \cdot 3$ & $11 \cdot 3$ & \\
\hline SD & $3 \cdot 0$ & 3.0 & $2 \cdot 9$ & 2.9 & \\
\hline Female (\%) & $47 \cdot 8$ & 54.5 & 51.4 & $45 \cdot 0$ & 0.3552 \\
\hline Urban (\%) & $29 \cdot 7$ & 30.8 & $31 \cdot 1$ & $30 \cdot 9$ & 0.7246 \\
\hline Overweight (\%) & $9 \cdot 1$ & $8 \cdot 1$ & $12 \cdot 5$ & 12.5 & 0.0558 \\
\hline Obesity (\%) & $5 \cdot 6$ & $9 \cdot 0$ & 6.5 & $12 \cdot 2$ & 0.0130 \\
\hline \multicolumn{6}{|l|}{ Traditional south } \\
\hline Age (years) & & & & & $<0.0001$ \\
\hline Mean & $10 \cdot 5$ & $11 \cdot 1$ & 11.4 & $12 \cdot 0$ & \\
\hline SD & $3 \cdot 0$ & $2 \cdot 8$ & $2 \cdot 9$ & $2 \cdot 8$ & \\
\hline Female (\%) & $52 \cdot 8$ & $55 \cdot 1$ & $48 \cdot 6$ & $42 \cdot 2$ & 0.0021 \\
\hline Urban (\%) & $32 \cdot 8$ & $30 \cdot 2$ & $26 \cdot 5$ & $33 \cdot 1$ & 0.8073 \\
\hline Overweight (\%) & $12 \cdot 5$ & 7.5 & $9 \cdot 3$ & $12 \cdot 8$ & 0.7141 \\
\hline Obesity (\%) & $8 \cdot 1$ & $9 \cdot 3$ & $7 \cdot 2$ & $8 \cdot 8$ & 0.9640 \\
\hline
\end{tabular}

* $P$ for trend was calculated from a linear regression analysis for continuous variables and Mantel-Haenszel $\chi^{2}$ for categorical variables. 
Table 3. Total energy and nutrient intakes across quartiles $(Q)$ of three dietary patterns in Chinese childrent (Mean values with their standard errors)

\begin{tabular}{|c|c|c|c|c|c|c|c|c|}
\hline & \multicolumn{8}{|c|}{ Quartile of dietary pattern score } \\
\hline & \multicolumn{2}{|c|}{ Q1 } & \multicolumn{2}{|c|}{ Q2 } & \multicolumn{2}{|c|}{ Q3 } & \multicolumn{2}{|c|}{ Q4 } \\
\hline & Mean & SEM & Mean & SEM & Mean & SEM & Mean & SEM \\
\hline \multicolumn{9}{|l|}{ Modern } \\
\hline Energy $(\mathrm{kJ})^{*}$ & $5998 \cdot 2$ & $137 \cdot 1$ & $6710 \cdot 3$ & $136 \cdot 3$ & $7297 \cdot 8$ & $136 \cdot 4$ & $8377 \cdot 8$ & $137 \cdot 3$ \\
\hline Carbohydrate (\% energy) ${ }^{\star}$ & $56 \cdot 2$ & 0.6 & 51.7 & 0.6 & $48 \cdot 1$ & 0.6 & $45 \cdot 2$ & 0.7 \\
\hline Fat $(\% \text { energy })^{\star}$ & $32 \cdot 9$ & 0.7 & $36 \cdot 2$ & 0.7 & 38.5 & 0.7 & $39 \cdot 1$ & 0.7 \\
\hline Fibre $(\mathrm{g})^{*}$ & 8.4 & 0.3 & 8.7 & 0.3 & $9 \cdot 1$ & 0.3 & $11 \cdot 1$ & 0.3 \\
\hline Vitamin C $(\mathrm{mg})^{\star}$ & 71.5 & 3.5 & 60.4 & 3.5 & $60 \cdot 2$ & 3.5 & $58 \cdot 2$ & 3.6 \\
\hline Vitamin A (retinol equivalents) ${ }^{\star}$ & 693.0 & $49 \cdot 4$ & 673.3 & $48 \cdot 1$ & 558.5 & $48 \cdot 0$ & $762 \cdot 0$ & 49.9 \\
\hline $\mathrm{Ca}(\mathrm{mg})^{\star}$ & $285 \cdot 8$ & $10 \cdot 6$ & 278.4 & $10 \cdot 3$ & 349.0 & $10 \cdot 3$ & $497 \cdot 3$ & $10 \cdot 7$ \\
\hline $\mathrm{Fe}(\mathrm{mg})^{\star}$ & $16 \cdot 5$ & 0.5 & $16 \cdot 7$ & 0.5 & $17 \cdot 2$ & 0.5 & $19 \cdot 2$ & 0.5 \\
\hline \multicolumn{9}{|l|}{ Traditional north } \\
\hline Energy $(\mathrm{kJ})^{\star}$ & $7368 \cdot 1$ & 143.4 & $6721 \cdot 8$ & $143 \cdot 3$ & 6794.4 & $143 \cdot 1$ & $7501 \cdot 6$ & $143 \cdot 5$ \\
\hline Carbohydrate (\% energy) ${ }^{\star}$ & $46 \cdot 2$ & 0.6 & $48 \cdot 0$ & 0.6 & $50 \cdot 3$ & 0.6 & $56 \cdot 7$ & 0.6 \\
\hline Fat $(\% \text { energy })^{\star}$ & $40 \cdot 1$ & 0.6 & $39 \cdot 1$ & 0.6 & $37 \cdot 0$ & 0.6 & 30.5 & 0.6 \\
\hline Fibre $(g)^{\star}$ & 8.0 & 0.3 & 8.4 & 0.3 & $9 \cdot 3$ & 0.3 & 11.6 & 0.3 \\
\hline Vitamin $\mathrm{C}(\mathrm{mg})^{\star}$ & $60 \cdot 7$ & 3.5 & $56 \cdot 7$ & 3.5 & $59 \cdot 1$ & 3.5 & $74 \cdot 0$ & 3.5 \\
\hline Vitamin A (retinol equivalents) ${ }^{\star}$ & $851 \cdot 7$ & $47 \cdot 7$ & $692 \cdot 1$ & $47 \cdot 7$ & $640 \cdot 5$ & $47 \cdot 6$ & $502 \cdot 1$ & $47 \cdot 8$ \\
\hline $\mathrm{Ca}(\mathrm{mg})$ & 373.2 & $11 \cdot 3$ & $343 \cdot 8$ & $11 \cdot 3$ & $355 \cdot 8$ & $11 \cdot 3$ & $337 \cdot 6$ & $11 \cdot 3$ \\
\hline $\mathrm{Fe}(\mathrm{mg})^{\star}$ & $16 \cdot 0$ & 0.5 & $16 \cdot 9$ & 0.5 & $18 \cdot 2$ & 0.5 & 18.4 & 0.5 \\
\hline \multicolumn{9}{|l|}{ Traditional south } \\
\hline Energy $(\mathrm{kJ})^{\star}$ & $6103 \cdot 7$ & $136 \cdot 2$ & $6447 \cdot 2$ & $135 \cdot 0$ & $7270 \cdot 8$ & 134.8 & 8563.5 & $136 \cdot 5$ \\
\hline Carbohydrate (\% energy) & $51 \cdot 3$ & 0.7 & $49 \cdot 1$ & 0.7 & $49 \cdot 8$ & 0.7 & $50 \cdot 9$ & 0.7 \\
\hline Fat $(\% \text { energy })^{\star}$ & $37 \cdot 0$ & 0.7 & $38 \cdot 2$ & 0.7 & $36 \cdot 9$ & 0.7 & $34 \cdot 7$ & 0.7 \\
\hline Fibre $(g)^{\star}$ & $8 \cdot 2$ & 0.3 & 8.7 & 0.3 & $9 \cdot 0$ & 0.3 & $11 \cdot 3$ & 0.3 \\
\hline Vitamin C $(\mathrm{mg})^{\star}$ & 41.3 & 3.4 & 52.4 & 3.4 & $62 \cdot 9$ & 3.3 & 93.8 & 3.5 \\
\hline Vitamin A (retinol equivalents) ${ }^{\star}$ & 394.5 & $48 \cdot 0$ & 594.1 & $47 \cdot 0$ & 664.5 & $46 \cdot 6$ & 1033.6 & $49 \cdot 3$ \\
\hline $\mathrm{Ca}(\mathrm{mg})^{\star}$ & $324 \cdot 0$ & 11.5 & 342.5 & $11 \cdot 3$ & 347.7 & $11 \cdot 2$ & $396 \cdot 2$ & 11.8 \\
\hline $\mathrm{Fe}(\mathrm{mg})^{*}$ & $15 \cdot 4$ & 0.5 & $16 \cdot 7$ & 0.5 & $17 \cdot 0$ & 0.5 & 20.5 & 0.5 \\
\hline
\end{tabular}

* Mean value was significantly different $(P<0.05)$.

$\dagger$ Adjusted for age and sex for energy and age, sex and total energy for other nutrients.

for confounders, children in the highest quartiles of the modern pattern score were more likely to be obese (OR $3 \cdot 10,95 \%$ CI $1 \cdot 52,6 \cdot 32)$. The likelihood of being obese was higher among those in the upper quartile compared to the lowest quartile (OR 2.42, $95 \%$ CI 1.34, 4.39) within the northern pattern. The OR for obesity was significantly higher according to quartile categories for the modern pattern ( $P$ for trend $=0.0024)$ and the traditional north pattern $(P$ for trend $=0.0060$ ) after adjustments for age, sex, living area, physical activity, annual household income per family member, and energy intake. However, there was no significant association between the southern pattern and obesity.

\section{Discussion}

We identified three distinct dietary patterns: the modern pattern, a traditional north pattern, and a traditional south pattern. The present study found that modern and northern patterns were positively associated with the risks of obesity after adjustments for putative risk factors. These results suggest that such dietary patterns are independently associated with obesity in Chinese children and adolescents.

It is difficult to compare these findings with earlier studies due to differing cultural and geographic factors and study methods ${ }^{(30)}$. However, the patterns we identified showed some similarities with other studies among children and adolescents. For example, the modern pattern is comprised of more energy and fat. Dietary fat provides the greatest amount of energy per g, foods high in fat are generally high in energy density, and so the modern pattern represented an energy-dense diet. A review recently concluded that energydense diets are a risk factor for obesity in children ${ }^{(31)}$. Some other studies have reported similar dietary patterns characterized by high intakes of energy and fat, such as the western, snacking, and junk/convenience patterns, even though the food items comprising these patterns were different ${ }^{(32)}$.

The modern pattern identified in the present study had high loadings mostly for convenience/snacking foods, including milk, fast foods, and eggs. Since 2004, a marked transition in snacking behaviors and patterns has occurred, with significant increases in the prevalence of snacking, the number of snacking occurrences in a day, and the contribution of snacks to total energy intake. Chinese children and adolescents are experiencing a dramatic increase in snacking ${ }^{(33)}$.

Milk consumption has been observed to be inversely associated with body mass or fat mass in cross-sectional studies of children and adolescents ${ }^{(34,35)}$. But we found that children in the highest quartiles of the modern pattern tended to be obese compared to their counterparts in the lowest quartile. It may be due to the other food items of this pattern, such as fast foods. Although more research needs to be conducted specifically in regard to the effects of fast foods on health, 
Table 4. Multivariate linear regression model to evaluate the effect of dietary pattern scores on BMI in Chinese children*

( $\beta$ Coefficients and $95 \%$ confidence intervals)

\begin{tabular}{lccc}
\hline & \multicolumn{3}{c}{$\mathrm{BMI}\left(\mathrm{kg} / \mathrm{m}^{2}\right)$} \\
\cline { 2 - 4 } & $\beta$ & $95 \% \mathrm{Cl}$ & $P$ \\
\hline Modern pattern score & & & \\
$\beta$ (continuous) & 0.59071 & $0.38966,0.79177$ & $<0.0001$ \\
Q1 & 0 & - & - \\
Q2 & 0.68601 & $0.15792,1.21411$ & 0.0109 \\
Q3 & 1.18812 & $0.64498,1.73125$ & $<0.0001$ \\
Q4 & 1.60862 & $1.04172,2.17552$ & $<0.0001$ \\
Traditional north & & & \\
pattern score & & & \\
$\beta$ (continuous) & 0.26592 & $0.07928,0.45257$ & 0.0053 \\
Q1 & 0 & - & - \\
Q2 & 0.11258 & $-0.41087,0.63604$ & 0.6731 \\
Q3 & 0.21900 & $-0.30421,0.74221$ & 0.4117 \\
Q4 & 1.08382 & $0.55900,1.60863$ & $<0.0001$ \\
Traditional south & & & \\
pattern score & & - & \\
$\beta$ (continuous) & 0.09225 & $-0.09783,0.28232$ & 0.3412 \\
Q1 & 0 & - & - \\
Q2 & 0.39597 & $-0.13190,0.92385$ & 0.1414 \\
Q3 & 0.04476 & $-0.57473,0.48522$ & 0.8684 \\
Q4 & 0.3312 & $-0.20536,0.86776$ & 0.2261
\end{tabular}

Q, quartile.

*Adjusted for age (continuous) and sex (male/female), living area (urban/rural), annual household income per family member (continuous), physical activity (continuous).

public health recommendations presently include the need to limit fast food consumption ${ }^{(36)}$.

The northern and the southern patterns had strong positive correlations with foods that are traditional in China. In the north of China, people are more likely to eat foods made from wheat flour like noodles and buns. However, the people in southern China prefer dishes with rice, vegetables, and pork. We therefore labeled these two patterns as 'traditional patterns' that were similar to the dietary patterns of Chinese adults ${ }^{(37)}$. Besides, the prevalence size of obesity varies from region to region in China. Our analysis showed that the prevalence of obesity was 13.9 and $5.8 \%$ in the north and south, respectively.

Following a traditional north consumption pattern, children in the upper quartile had a significantly higher likelihood of being obese compared to the lowest quartile. Compared with the staple food of rice, wheat absorbs less water when cooked $^{(38)}$. So the energy density of the traditional north pattern is higher than the traditional south pattern. It shows that high energy-dense diets are related to obesity ${ }^{(39)}$.

One characteristic of the northern pattern is high carbohydrate intake. To date, the mechanism underlying the carbohydrate-induced risk for obesity is still unclear. It is likely that carbohydrate intake may alter lipid profiles, such as an increase in TAG and/or a decrease in HDL-cholesterol, leading to increased obesity ${ }^{(40)}$. Further studies are needed to elucidate a detailed mechanism.

In China, the northern diet is simple and has less diversity, mainly including wheat noodles, dumplings, steamed buns and flat cakes. So the northern pattern included lower levels of micronutrients, that is, it represented a low nutrient-dense diet. Micronutrients like Fe, vitamin C, and vitamin A may play an important role in fat deposition and the pathogenesis of obesity ${ }^{(41)}$. Low nutrient-dense diets appear to favour the development of central nervous system insulin resistance, which might in part be responsible for leptin resistance, which, accordingly, promotes pleasurable responses to foods ${ }^{(42,43)}$. However, the association between dietary patterns and obesity in the present study cannot be fully explained by the nutrient-density issue.

We are aware that the presesnt study has several limitations. First, the results do not show the 'cause and effect' relationship between dietary patterns and risk of obesity, as the investigation is based upon cross-sectional data. Second, the statistical methods used to define the dietary patterns are somewhat subjective, including the consolidation of food items into food groups, the number of factors to extract, and the labelling of the pattern ${ }^{(44)}$. Third, dietary patterns could vary among studies because of diverse ethnicities/cultures or

Table 5. Association of dietary patterns with childhood obesity in China (Odds ratios and $95 \%$ confidence intervals)

\begin{tabular}{|c|c|c|c|c|c|c|c|c|}
\hline & \multicolumn{8}{|c|}{ Quartile of dietary pattern score } \\
\hline & \multirow[b]{2}{*}{ Q1 } & \multicolumn{2}{|c|}{ Q2 } & \multicolumn{2}{|c|}{ Q3 } & \multicolumn{2}{|c|}{ Q4 } & \multirow[b]{2}{*}{$P$ for trend } \\
\hline & & OR & $95 \% \mathrm{Cl}$ & OR & $95 \% \mathrm{Cl}$ & OR & $95 \% \mathrm{Cl}$ & \\
\hline \multicolumn{9}{|l|}{ Modern } \\
\hline Model $1^{\star}$ & 1.00 & 1.94 & $0.98,3.84$ & $2 \cdot 13$ & $1.07,4.24$ & 3.55 & $1.80,7.03$ & 0.0003 \\
\hline Model $2 \dagger$ & 1.00 & 1.85 & $0.93,3.69$ & 1.98 & $0.98,3.97$ & $3 \cdot 10$ & $1.52,6.32$ & 0.0024 \\
\hline \multicolumn{9}{|c|}{ Traditional north } \\
\hline Model 1 & 1.00 & 1.64 & $0.88,3.05$ & $1 \cdot 16$ & $0.60,2 \cdot 24$ & 2.45 & $1.35,4.44$ & 0.0038 \\
\hline Model 2 & 1.00 & 1.75 & $0.94,3.27$ & 1.21 & $0.62,2.35$ & 2.42 & $1.34,4.39$ & 0.0060 \\
\hline \multicolumn{9}{|c|}{ Traditional south } \\
\hline Model 1 & 1.00 & 1.36 & $0.78,2.39$ & 1.04 & $0.57,1.89$ & 1.44 & $0.81,2.57$ & 0.3413 \\
\hline Model 2 & 1.00 & 1.32 & $0.75,2.33$ & 0.93 & $0.51,0.71$ & $1 \cdot 15$ & $0.62,2.11$ & 0.8976 \\
\hline
\end{tabular}

Q, quartile.

* Model 1: adjusted for age (continuous) and sex (male/female), living area (urban/rural), annual household income per family member (continuous), physical activity (continuous).

† Model 2: Model 1 additionally adjusted for total energy intake (continuous). 
objectives of study. It is difficult to compare findings of the present study with those of others. Fourth, the 24-h dietary recall method cannot reliably evaluate usual dietary intake.

Despite these limitations, this is the first study to reveal the relationships between dietary patterns and risk of obesity in Chinese children and adolescents, using large survey data. In 2011, the three largest municipal cities (Beijing, Shanghai, and Chongqing) were added to the study. Thus, the 2011survey included twelve primary sample units consisting of individual provinces or autonomous cities. Because of the greater inclusivity of the data, the present study was useful in providing a better understanding of the dietary habits of the children and adolescents in China.

\section{Conclusion}

The present study indicates that childhood obesity is associated with dietary patterns in China; in particular, the modern dietary pattern and traditional north dietary pattern were positively associated with obesity risk. These findings, we hope, will be of some help in developing future childhood obesity interventions. However, further studies are needed to understand more objectively the relationship between dietary patterns and obesity using prospective data.

\section{Acknowledgements}

This research was supported in part by the National Institutes of Health (NIH), by grant number U54HD070725 from the Eunice Kennedy Shriver National Institute of Child Health \& Human Development (NICHD). The U54 project is COfunded by the NICHD and the NIH Office of Behavioral and Social Sciences Research (OBSSR). The NIH U54 center grant funded three key research projects including one in China, the only international project, and Y. W. is the principal investigator (PI) and H. W. is the CO-PI. H. W. is the original PI of the U54 grant. The present research used data collected in the CHNS. The authors are grateful to the participants for their involvement in the survey. The authors also thank the team at National Institute for Nutrition and Health, Chinese Center for Disease Control and Prevention, and the Carolina Population Center, University of North Carolina at Chapel Hill. The CHNS data collection was in part funded by research grants from the NIH (R01-HD30880, DK056350, R24 HD050924, and R01-HD38700) and the NIH Fogarty International Center. The content of the paper is solely the responsibility of the authors and does not necessarily represent the official views of the funders.

The authors declare that there are no conflicts of interest.

The authors' contributions are as follows: J. G. Z. conducted data collection, data management, statistical analysis and interpretation, manuscript design and writing. H. W. conducted data collection and data management, and advised on statistical analysis. F. Z. was primarily responsible for the supervision of the CHNS. Y. W. and H. X. contributed to data analysis and critical revisions of the manuscript. Z. W., W. D. and C. S. contributed to data collection and editing of the final version of the manuscript. J. Z. and H. J. revised the manuscript. B. Z. the corresponding author, had the primary responsibility for the final content. All authors read and approved the final manuscript.

\section{References}

1. Karnik S \& Kanekar A (2012) Childhood obesity: a global public health crisis. Int J Prev Med 3, 1-7.

2. Gupta N, Goel K, Shah P, et al. (2012) Childhood obesity in developing countries: epidemiology, determinants, and prevention. Endocr Rev 33, 48-70.

3. Wang Y \& Lobstein T (2006) Worldwide trends in childhood overweight and obesity. Int J Pediatr Obes 1, 11-25.

4. Nguyen DM \& El-Serag HB (2010) The epidemiology of obesity. Gastroenterol Clin North Am 39, 1-7.

5. Barton M (2012) Childhood obesity: a life-long health risk. Acta Pharmacol Sin 33, 189-193.

6. Wang Y, Bentley ME, Zhai F, et al. (2002) Tracking of dietary intake patterns of Chinese from childhood to adolescence over a six-year follow-up period. J Nutr 132, 430-438.

7. Popkin BM, Adair LS \& Ng SW (2012) Global nutrition transition and the pandemic of obesity in developing countries. Nutr Rev 70, 3-21.

8. Bray GA, Paeratakul S \& Popkin BM (2004) Dietary fat and obesity: a review of animal, clinical and epidemiological studies. Physiol Behav 83, 549-555.

9. Boeing H, Bechthold A, Bub A, et al. (2012) Critical review: vegetables and fruit in the prevention of chronic diseases. Eur J Nutr 51, 637-663.

10. Anderson JW, Baird P, Davis RH, et al. (2009) Health benefits of dietary fiber. Nutr Rev 67, 188-205.

11. Hu FB (2002) Dietary pattern analysis: a new direction in nutritional epidemiology. Curr Opin Lipidol 13, 3-9.

12. Van Horn L (2011) Eating pattern analyses: the whole is more than the sum of its parts. J Am Diet Assoc 111, 203.

13. Tucker KL (2010) Dietary patterns, approaches, and multicultural perspective. Appl Physiol Nutr Metab 35, 211-218.

14. Kant AK (2004) Dietary patterns and health outcomes. J Am Diet Assoc 104, 615-635.

15. Popkin BM (2014) Synthesis and implications: China's nutrition transition in the context of changes across other low- and middle-income countries. Obes Rev 15, Suppl. 1, 60-67.

16. Dearth-Wesley T, Wang H \& Popkin BM (2008) Under- and overnutrition dynamics in Chinese children and adults (1991-2004). Eur J Clin Nutr 62, 1302-1307.

17. Wang Y, Popkin B \& Zhai F (1998) The nutritional status and dietary pattern of Chinese adolescents, 1991 and 1993. Eur J Clin Nutr 52, 908-916.

18. Yu Z, Han S, Chu J, et al. (2012) Trends in overweight and obesity among children and adolescents in China from 1981 to 2010: a meta-analysis. PLOS ONE 7, e51949.

19. Gordon-Larsen P, Wang H \& Popkin BM (2014) Overweight dynamics in Chinese children and adults. Obes Rev $\mathbf{1 5}$, Suppl. 1, 37-48.

20. Shin KO, Oh SY \& Park HS (2007) Empirically derived major dietary patterns and their associations with overweight in Korean preschool children. Br J Nutr 98, 416-421.

21. Song Y, Park MJ, Paik HY, et al. (2010) Secular trends in dietary patterns and obesity-related risk factors in Korean adolescents aged 10-19 years. Int J Obes (Lond) 34, 48-56.

22. Shroff MR, Perng W, Baylin A, et al. (2014) Adherence to a snacking dietary pattern and soda intake are related to the development of adiposity: a prospective study in schoolage children. Public Health Nutr 17, 1507-1513. 
23. Johnson L, Mander AP, Jones LR, et al. (2008) Energydense, low-fiber, high-fat dietary pattern is associated with increased fatness in childhood. Am J Clin Nutr 87, 846-854.

24. Oellingrath IM, Svendsen MV \& Brantsaeter AL (2010) Eating patterns and overweight in 9- to 10-year-old children in Telemark County, Norway: a cross-sectional study. Eur J Clin Nutr 64, 1272-1279.

25. Popkin BM, Du S, Zhai F, et al. (2009) Cohort Profile: The China Health and Nutrition Survey - monitoring and understanding socio-economic and health change in China, 1989-2011. Int J Epidemiol 39, 1435-1440.

26. Zhai FY, Du SF, Wang ZH, et al. (2014) Dynamics of the Chinese diet and the role of urbanicity, 1991-2011. Obes Rev 15, Suppl. 1, 16-26.

27. WHO (1995) Physical Status: The Use and Interpretation of Anthropometry. Report of a WHO Expert Committee. World Health Organization Technical Report Series no. 854, pp. 1-452. Geneva: WHO.

28. Group of China Obesity Task Force (2004) [Body mass index reference norm for screening overweight and obesity in Chinese children and adolescents]. Zhonghua Liu Xing Bing Xue Za Zhi 25, 97-102.

29. Kim J-O MC (1978) Factor Analysis: Statistical Methods and Practical Issues. Thousand Oaks, CA: Sage Publications.

30. Lioret S, Touvier M, Lafay L, et al. (2008) Dietary and physical activity patterns in French children are related to overweight and socioeconomic status. J Nutr 138, 101-107.

31. Perez-Escamilla R, Obbagy JE, Altman JM, et al. (2012) Dietary energy density and body weight in adults and children: a systematic review. J Acad Nutr Diet 112, 671-684.

32. Ambrosini GL (2014) Childhood dietary patterns and later obesity: a review of the evidence. Proc Nutr Soc 73, $137-146$

33. Wang Z, Zhai F, Zhang B, et al. (2012) Trends in Chinese snacking behaviors and patterns and the social-demographic role between 1991 and 2009. Asia Pac J Clin Nutr 21, 253-262.

34. Barba G, Troiano E, Russo P, et al. (2005) Inverse association between body mass and frequency of milk consumption in children. Br J Nutr 93, 15-19.

35. Novotny R, Daida YG, Acharya S, et al. (2004) Dairy intake is associated with lower body fat and soda intake with greater weight in adolescent girls. J Nutr 134, 1905-1909.

36. Rosenheck R (2008) Fast food consumption and increased caloric intake: a systematic review of a trajectory towards weight gain and obesity risk. Obes Rev 9, 535-547.

37. Wang D, He Y, Li Y, et al. (2011) Dietary patterns and hypertension among Chinese adults: a nationally representative cross-sectional study. BMC Public Health 11, 925.

38. Shi Z, Taylor AW, Hu G, et al. (2012) Rice intake, weight change and risk of the metabolic syndrome development among Chinese adults: the Jiangsu Nutrition Study (JIN). Asia Pac J Clin Nutr 21, 35-43.

39. Prentice AM \& Jebb SA (2003) Fast foods, energy density and obesity: a possible mechanistic link. Obes Rev 4, 187-194.

40. Mittendorfer B \& Sidossis LS (2001) Mechanism for the increase in plasma triacylglycerol concentrations after consumption of short-term, high-carbohydrate diets. Am J Clin Nutr 73, 892-899.

41. Garcia OP, Ronquillo D, Caamano Mdel C, et al. (2012) Zinc, vitamin $\mathrm{A}$, and vitamin $\mathrm{C}$ status are associated with leptin concentrations and obesity in Mexican women: results from a cross-sectional study. Nutr Metab (Lond) 9, 59.

42. Isganaitis E \& Lustig RH (2005) Fast food, central nervous system insulin resistance, and obesity. Arterioscler Thromb Vasc Biol 25, 2451-2462.

43. Garcia OP, Long KZ \& Rosado JL (2009) Impact of micronutrient deficiencies on obesity. Nutr Rev 67, 559-572.

44. Martinez ME, Marshall JR \& Sechrest L (1998) Invited Commentary: factor analysis and the search for objectivity. Am J Epidemiol 148, 17-19.

Appendix 1. Food groups in the factor analysis

\begin{tabular}{ll}
\hline Food or food groups & Foods included in the group \\
\hline Rice & Round-grained rice, long-grained rice, glutinous rice \\
Wheat & Wheat bun, wheat noodles \\
Other cereals & Maize, barley, millet \\
Tubers & Potato, sweet potato \\
Legumes & Soyabeans, and products \\
Fungi and algae & Mushroom, kelp, laver \\
Vegetables & Cabbage, eggplant, carrot, pepper, lettuce, rape, tomato, cauliflower \\
Fruits & Apple, pear, peach, date, grape, watermelon, orange, other fruit \\
Pork & Pork and pork products \\
Other livestock meat & Beef, game, lamb, meat products \\
Poultry & Chicken, duck, goose \\
Organ meat & Organ meat \\
Aquatic products & Fish, shrimp, crab, shellfish \\
Milk & Milk and products \\
Eggs & Eggs \\
Nuts & Nuts \\
Sugary foods & Beet sugar, honey \\
Cake & Cake, ethnic foods \\
Fast foods & Convenience food, hamburger, pizza, sandwich, French fries \\
Beverages & Carbonated drinks, fruit juice, vegetable juice
\end{tabular}

